\title{
In Vivo Measurement of Glenohumeral Joint Contact Patterns
}

\author{
Michael J. Bey, Stephanie K. Kline, Roger Zauel, Patricia A. Kolowich, and Terrence R. Lock
}

Department of Orthopaedic Surgery, Bone and Joint Center, Henry Ford Hospital, 2799 W. Grand Blvd., E\&R 2015, Detroit, MI 48202, USA

Correspondence should be addressed to Michael J. Bey, bey@bjc.hfh.edu

Received 15 April 2009; Accepted 27 June 2009

Academic Editor: João Manuel R. S. Tavares

Copyright ( $) 2010$ Michael J. Bey et al. This is an open access article distributed under the Creative Commons Attribution License, which permits unrestricted use, distribution, and reproduction in any medium, provided the original work is properly cited.

\begin{abstract}
The objectives of this study were to describe a technique for measuring in-vivo glenohumeral joint contact patterns during dynamic activities and to demonstrate application of this technique. The experimental technique calculated joint contact patterns by combining CT-based 3D bone models with joint motion data that were accurately measured from biplane x-ray images. Joint contact patterns were calculated for the repaired and contralateral shoulders of 20 patients who had undergone rotator cuff repair. Significant differences in joint contact patterns were detected due to abduction angle and shoulder condition (i.e., repaired versus contralateral). Abduction angle had a significant effect on the superior/inferior contact center position, with the average joint contact center of the repaired shoulder $12.1 \%$ higher on the glenoid than the contralateral shoulder. This technique provides clinically relevant information by calculating in-vivo joint contact patterns during dynamic conditions and overcomes many limitations associated with conventional techniques for quantifying joint mechanics.
\end{abstract}

\section{Introduction}

The treatment of many pathologic shoulder conditions (e.g., rotator cuff tears, glenohumeral joint instability) relies implicitly on the belief that restoring normal glenohumeral joint mechanics is necessary to obtain a satisfactory clinical result. However, the measurement of glenohumeral joint mechanics-in particular, the patterns of contact between the humerus, and glenoid-has been a challenging task, especially under in vivo conditions. Previous research has measured glenohumeral joint mechanics under in-vitro conditions with cadaveric specimens (e.g., [1-3]), and under in vivo conditions with standard clinical imaging techniques such as magnetic resonance imaging (MRI) [48], fluoroscopy [9-12], and computed tomography (CT) [13]. However, there are limitations associated with these conventional measuring techniques. Specifically, cadaveric studies cannot accurately simulate in vivo conditions because muscle forces and joint forces are unknown. MRI and CT are largely restricted to acquiring images under static conditions and conventional fluoroscopy is not designed to accurately measure motion in three dimensions. Thus, these conventional measurement techniques were not designed to assess three-dimensional, in vivo glenohumeral joint contact patterns during dynamic activities. Therefore, the objectives of this study are to (1) describe a technique for measuring in vivo glenohumeral joint contact patterns during dynamic activities, and (2) demonstrate application of this technique by characterizing differences between shoulders in patients who had undergone rotator cuff repair.

\section{Methods}

2.1. Subjects. Following institutional review board approval and informed consent, 20 subjects $(13$ males, 7 females; age: $65.1 \pm 10.4$ ) enrolled in the study. Each subject had arthroscopic surgical repair of an isolated supraspinatus tendon tear approximately 4 months prior to participating in the study. All tears were directly repaired to bone using a double row technique [14] and an anterior acromioplasty was also performed. Each patient's shoulder was placed in a sling postoperatively. Active motion exercises were initiated at six weeks postsurgery, and progressive resistance training was initiated at 10-12 weeks postsurgery. The contralateral shoulder of each subject was asymptomatic, with no history of shoulder injury or surgery.

2.2. Testing Setup. Subjects were positioned with their shoulder centered within a biplane X-ray system [15]. The system consists of two $100 \mathrm{~kW}$ pulsed X-ray generators (EMD 
Technologies CPX 3100CV, Quebec, Canada) and two $30 \mathrm{~cm}$ image intensifiers (Shimadzu AI5765HVP, Kyoto, Japan), optically coupled to synchronized high-speed video cameras (Phantom IV, Vision Research, Wayne, NJ, USA), configured in a custom gantry to enable a variety of motion studies. Subjects wore a lead-lined thyroid shield and protective vest during testing to minimize X-ray exposure.

2.3. Testing Procedures. Glenohumeral joint motion was assessed by tracking the $3 \mathrm{D}$ position of the humerus and scapula from images acquired from the biplane $\mathrm{X}$ ray system. Images were acquired at $60 \mathrm{~Hz}$ with the $\mathrm{X}$ ray generators in pulsed mode while subjects performed coronal-plane abduction. Subjects began this motion with their arm in a fully adducted neutral-rotation position, resting comfortably at their side. The ending position for this task was approximately $120^{\circ}$ of humerothoracic motion, that is, the angle formed between the humerus and the torso. Subjects performed this motion while holding a 3-pound hand weight, or a weight less than this that was consistent with the patient's stage of rehabilitation. Subjects were instructed to perform this motion at a frequency of $0.25 \mathrm{~Hz}$, so that one complete motion cycle took four seconds. The rate of shoulder motion was controlled using a metronome. Subjects performed three trials with a minimum of three minutes between trials to minimize fatigue. In addition, biplane X-ray images were acquired for a single static trial at the starting position. This static trial served as a reference position to which all glenohumeral joint motion data were compared. Both the repaired and contralateral shoulders were tested and the testing order was randomized.

Following testing, bilateral CT scans of the entire humerus and scapula were acquired (GE Medical Systems, LightSpeed16, Piscataway, NJ, USA). The scans were performed in axial mode with a slice thickness of $1.25 \mathrm{~mm}$ and an in-plane resolution of approximately $0.5 \mathrm{~mm}$ per pixel. The humerus and scapula were isolated from other bones and soft tissue using a semiautomatic segmentation technique (Mimics 10.1, Materialise, Leuven, Belgium). The CT volume was then interpolated using a feature-based interpolation technique and scaled to have cubic voxels with dimensions similar to the $2 \mathrm{D}$ pixel size in the biplane $\mathrm{X}$-ray images.

2.4. Measuring Glenohumeral Joint Motion. The 3D position and orientation of the humerus and scapula were tracked from the biplane X-ray images using a 3D model-based tracking technique [16]. This technique uses a six degreeof-freedom optimization algorithm to find the best match between the biplane X-ray images and a pair of digitally reconstructed radiographs (DRRs) generated via ray-traced projection through the CT-based bone model. By optimizing the correlation between the two DRRs and the actual $2 \mathrm{D}$ biplane $\mathrm{X}$-ray image pairs, the in vivo position and orientation of a given bone can be estimated. This modelbased tracking technique has been shown to have an accuracy of better than $\pm 0.4 \mathrm{~mm}$ and $\pm 0.5^{\circ}$ for measuring in vivo shoulder motion during dynamic activities [16].

Transformations between each bone's $3 \mathrm{D}$ position and anatomical axes were determined from the CT-based bone

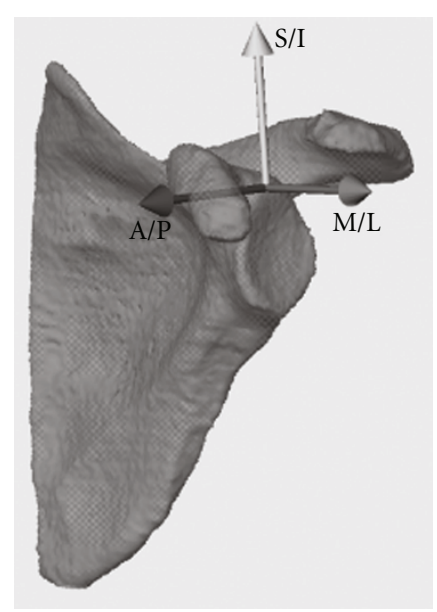

FIGURE 1: The contact center location was expressed relative to a subject-specific scapula coordinate system. The axes of the scapula coordinate system are aligned in the anterior/posterior ( $X$ axis), superior/inferior ( $Y$ axis), and medial/lateral ( $Z$ axis) directions.

models using custom software (based on Open Inventor 5.0, Mercury Computer Systems, Chelmsford, Mass, USA) that was developed to locate specific anatomical landmarks and construct standardized anatomical coordinate systems (Figure 1) [17]. To minimize side-to-side variability in kinematic measures due solely to anatomical axis locations, the same anatomical landmark locations identified on the humerus and scapula of the repaired shoulder were used for the contralateral shoulder. This was accomplished by mirrorimaging the contralateral shoulder CT-based bone models, manually coregistering these bone models with the repaired shoulder's CT-based bone models, and then transferring the anatomical landmark locations to the contralateral shoulder's CT-based bone models. Rotations of the humerus relative to the glenoid were calculated using a standard Euler angle sequence in which the first rotation defined the plane of elevation, the second rotation described the amount of elevation, and the third rotation represented the amount of internal/external rotation [18].

2.5. Measuring Glenohumeral Joint Contact Patterns. Glenohumeral joint contact patterns were determined by combining the joint motion measured from the biplane X-ray images with the subject-specific CT bone models. Briefly, the CT-based bone models were first converted into 3D surface models constructed of contiguous triangular tiles. A typical humerus or scapula model contained approximately 70000 triangles of $0.5 \mathrm{~mm}^{2}$ each. To avoid unnecessary calculation, two specific regions of interest were identified: the humeral head and the glenoid. After co-registering the surface models with the kinematic data, the custom software calculated the $3 \mathrm{D}$ distance from every surface-triangle centroid on the humeral head to every surface-triangle centroid on the glenoid (Figure 2(a)). The contact center location was then determined by calculating the centroid of the closest $200 \mathrm{~mm}^{2}$ region of contact between the humerus and glenoid (Figure 2(b)). The 3D coordinates of this contact center 


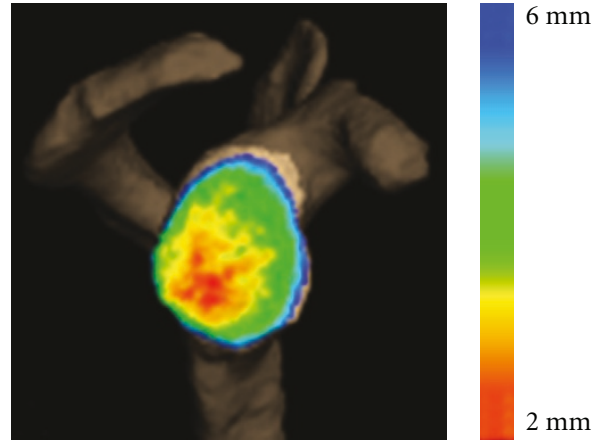

(a)

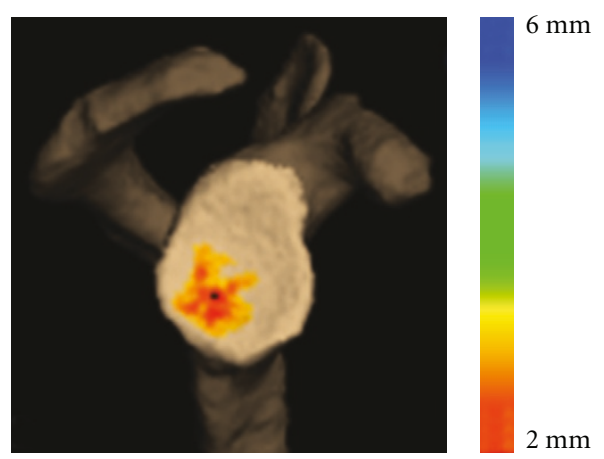

(b)

Figure 2: (a) Colormap of the minimum distance between the glenoid and humerus for a single frame of data. (b) The contact center location (indicated by the black dot) was calculated as the centroid of the closest $200 \mathrm{~mm}^{2}$ region between the humerus and glenoid.

location were then expressed relative to the scapula-based coordinate system, with the medial/lateral coordinate always located on the glenoid surface. This process was repeated for all frames of every trial. These calculations resulted in a $3 \mathrm{D}$ contact path, that is, a time-series of glenohumeral joint contact data at each point in time.

Due to differences in glenoid size between subjects, these glenohumeral joint contact data were normalized relative the size of each subject's glenoid. Specifically, we first used custom software developed in our laboratory to manually measure the glenoid's maximum superior/inferior $(\mathrm{S} / \mathrm{I})$ and maximum anterior/posterior $(\mathrm{A} / \mathrm{P})$ dimensions from the CT-based bone models. For each subject, the 3D joint contact center coordinates were then normalized by (1) dividing the $\mathrm{A} / \mathrm{P}$ contact center location by the maximum $\mathrm{A} / \mathrm{P}$ glenoid dimension, and (2) dividing the S/I contact center location by the maximum S/I glenoid dimension. Thus, the data were expressed as a percentage of the maximum glenoid dimensions in both the $\mathrm{A} / \mathrm{P}$ and $\mathrm{S} / \mathrm{I}$ directions. These normalized contact center position data were then averaged across subjects in $5^{\circ}$ increments from $10^{\circ}$ to $70^{\circ}$ of glenohumeral abduction.

2.6. Outcome Measures. To quantify differences in joint contact patterns between the repaired and contralateral shoulders, we calculated five outcome measures from the

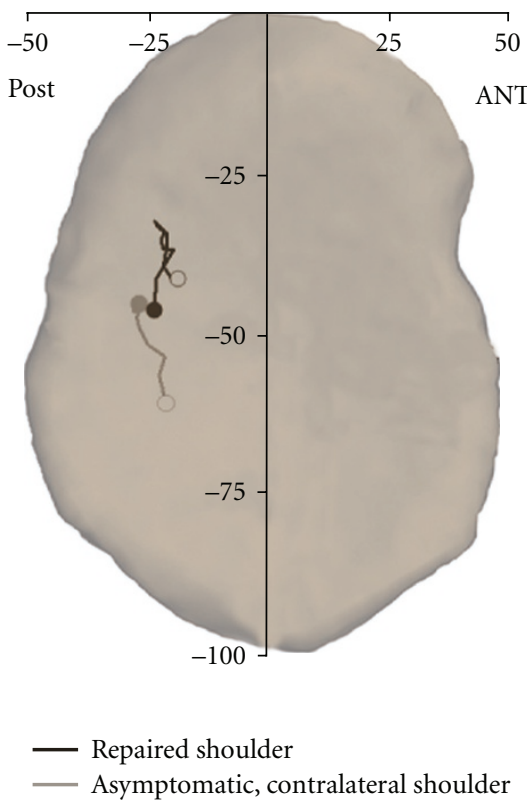

Figure 3: Average path of the glenohumeral joint contact center (superimposed on a typical glenoid) during coronal-plane abduction. For each path, the open circle $(\circ)$ indicates the starting position and the closed circle $(\bullet)$ indicates the ending position. ANT: anterior, POST: posterior.

normalized 3D contact center data. These outcome measures, averaged across all trials, included $\mathrm{A} / \mathrm{P}$ contact center position, S/I contact center position, A/P contact position range, S/I contact position range, and contact path length.

2.7. Statistical Analysis. We used a two-way repeated measures ANOVA to assess the effects of glenohumeral joint abduction angle (from $10^{\circ}$ to $70^{\circ}$ in $10^{\circ}$ increments) and shoulder condition (repaired versus contralateral) on the normalized A/P and S/I contact center position. The effect of shoulder condition (repaired versus contralateral) on average $\mathrm{A} / \mathrm{P}$ contact center position, average S/I contact center position, $\mathrm{A} / \mathrm{P}$ contact position range, $\mathrm{S} / \mathrm{I}$ contact position range, and contact path length was assessed with a paired $t$ test. Significance was set at $P<.05$.

\section{Results}

The experimental technique presented here was sufficiently sensitive to detect differences in joint contact patterns as a function of both abduction angle and shoulder condition (i.e., repaired versus contralateral). The joint contact center position moved predominantly in the S/I direction and relatively little in the $\mathrm{A} / \mathrm{P}$ direction during shoulder abduction in both the repaired and contralateral shoulders (Figure 3), with abduction angle having a significant effect on $\mathrm{S} / \mathrm{I}$ contact center position $(P=.004)$ but not $\mathrm{A} / \mathrm{P}$ contact center position $(P=.675)$. Interestingly, the path of the joint contact center changed direction during abduction in the repaired shoulders. Specifically, the joint contact center location moved superiorly on the glenoid 


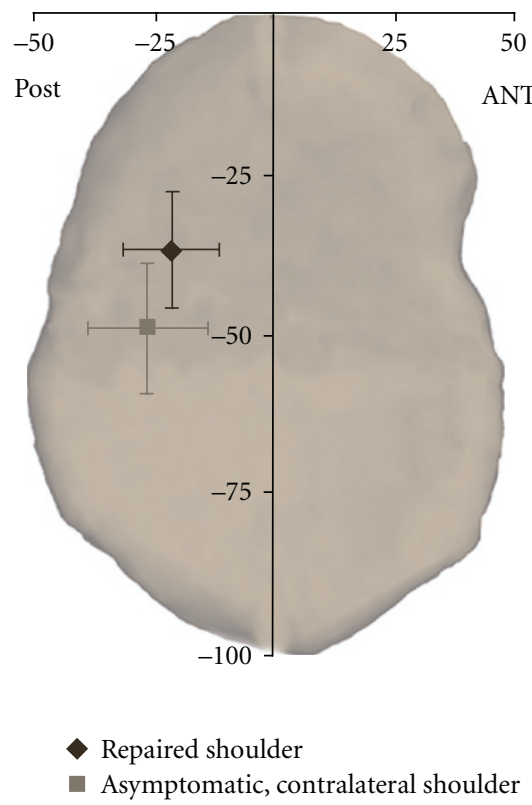

Figure 4: Average contact center position from $10^{\circ}$ to $70^{\circ}$ of coronal-plane abduction. Significant differences in both the average $\mathrm{S} / \mathrm{I}(P=.01)$ and $\mathrm{A} / \mathrm{P}(P=.04)$ contact center position were detected between the repaired and contralateral shoulders. ANT: anterior, POST: posterior.

from $10^{\circ}$ to $40^{\circ}$ of glenohumeral abduction, but then moved inferiorly on the glenoid from $40^{\circ}$ to $70^{\circ}$ of abduction (Figure 3). Consequently, the distance between the joint contact center locations associated with the repaired shoulders' starting position ( $10^{\circ}$ of glenohumeral abduction) and ending position $\left(70^{\circ}\right.$ of glenohumeral abduction) was only $1.5 \mathrm{~mm}$. In contrast, the distance between the joint contact center locations at the starting and ending positions in the contralateral shoulders was $5.4 \mathrm{~mm}$ as the joint contact center path did not change direction during abduction.

Shoulder condition (i.e., repaired versus contralateral) had a significant effect on both the S/I $(P<.001)$ and A/P $(P=.029)$ contact center position. Specifically, the repaired shoulders' average joint contact center was $12.1 \% \pm 6.4 \%$ higher on the glenoid $(P=.01)$ and $3.7 \% \pm 2.5 \%$ more anterior on the glenoid $(P=.04)$ than the contralateral shoulders' average joint contact center (Figure 4). However, the study did not detect statistically significant differences between the repaired and contralateral shoulders in terms of $\mathrm{A} / \mathrm{P}$ contact center range $(P=.18$, Figure 5$), \mathrm{S} / \mathrm{I}$ contact center range $(P=.10$, Figure 5$)$, or contact path length $(P=.89$, Figure 5$)$.

\section{Discussion}

This study describes a technique for measuring in vivo glenohumeral joint contact patterns during dynamic activities, and demonstrates application of this technique by characterizing differences between repaired and contralateral shoulders of patients who have undergone rotator cuff repair. The experimental method described here offers advantages

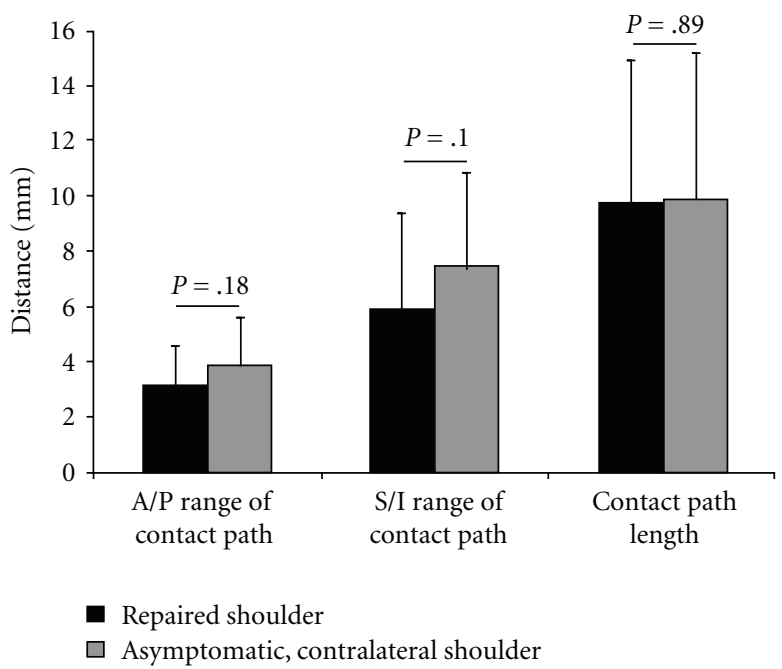

FIGURE 5: No statistically significant differences were detected between the repaired and contralateral shoulders in terms of $\mathrm{A} / \mathrm{P}$ contact center position range $(P=.18)$, S/I contact center position range $(P=.10)$, or contact path length $(P=.89)$.

over conventional techniques for describing glenohumeral joint motion. Specifically, glenohumeral joint contact patterns provide a measure of joint function that may not be adequately captured when reporting only conventional measures of humeral rotation and translation. This is important, since many pathologic conditions of the shoulder (e.g., rotator cuff tear, glenohumeral joint instability) are believed to alter the glenohumeral joint articular mechanics, and procedures for treating these common conditions rely implicitly on the belief that restoring normal glenohumeral joint mechanics is necessary to obtain a satisfactory outcome. The approach described here of quantifying joint contact patterns has also been used by other investigators as a means of detecting functional differences associated with a specific clinical condition (e.g., distal radius malunion [19, 20]) that can not be detected using conventional kinematic parameters. Thus, joint contact patterns are perhaps not only a more sensitive measurement than conventional kinematics for detecting subtle differences in joint function but may also provide a more clinically relevant indication of the extent to which a conservative or surgical procedure has adequately restored normal joint function.

Glenohumeral joint contact patterns have been quantified in a number of cadaveric studies. For example, the effects of shoulder position on glenohumeral joint contact patterns have been studied in cadaver specimens using stereophotogrammetry [21-23]. Soslowsky et al. indicated that the glenoid contact location was primarily in the anterior half of the glenoid with the shoulder adducted, but moved posteriorly with increasing abduction [23]. In contrast, the current study demonstrated that the contact center was always located on the posterior half of the glenoid (in both the repaired and contralateral shoulders), and that there was little change in the A/P contact center location with increasing abduction. Furthermore, while the current 
study demonstrated significant changes in the S/I contact center location with increasing abduction (Figure 3 ), the study by Soslowsky et al. reported no clear shift in the S/I direction in glenoid contact patterns with abduction. One plausible explanation that may help to reconcile these differences is that these previous cadaveric studies simulated scapular-plane abduction whereas the subjects in the current study elevated their shoulders in the coronal plane. Cadaveric studies have also investigated the effects of shoulder position, joint contact forces, muscles forces, and various simulated clinical conditions on joint contact area and joint contact pressures by inserting thin pressure-sensitive films or similar devices (e.g., Fuji film or Tekscan sensors) between the humerus and glenoid $[1-3,24,25]$. Although these types of cadaveric experiments have provided the bulk of existing knowledge about glenohumeral joint mechanics, cadaveric studies are not capable of accurately reproducing the complex muscle forces, joint forces, or joint motions that occur in vivo. Furthermore, given that rotator cuff disease typically develops slowly over many years, the inability to study biological response or disease progression is another significant limitation of cadaver studies.

One limitation of this technique for measuring joint contact patterns is that it neglects cartilage, since cartilage is difficult to image with both CT and conventional radiography. DeFrate and colleagues have suggested that neglecting cartilage could potentially lead to erroneous measures of joint contact in the knee due to variations in cartilage thickness across the femur and tibia [26]. Previous research has demonstrated that cartilage thickness varies with position on the glenoid and humeral head too, but that cartilage thickness has an inverse relationship between these articulating surfaces [27-30]. In particular, it has been shown that cartilage thickness for the humeral head is highest in the center and lowest at the periphery. In contrast, cartilage thickness on the glenoid is lowest in the center of the glenoid and higher at the periphery. The significance of this inverse relationship is that based on the data by Soslowsky and colleagues [27], the range of total cartilage thickness (i.e., the sum of glenoid cartilage thickness and humeral head cartilage thickness) over the regions of contact on the glenoid and humeral head during coronal-plane abduction varies by only $0.4 \mathrm{~mm}$. Since the range of total cartilage thickness is equal to the uncertainty associated with the model-based tracking technique $( \pm 0.4 \mathrm{~mm}[16])$, the current approach is not sufficiently accurate to detect changes in joint contact associated with subtle variations in total cartilage thickness. Thus, there is currently no advantage to including cartilage information in our subject-specific bone models. However, we anticipate additional technical enhancements will improve the accuracy of our model-based tracking technique, and therefore future efforts will focus on developing and validating (under conditions that provide a realistic simulation of in vivo testing conditions) a technique that includes cartilage in the estimation of joint contact patterns.

In summary, we have developed a technique for characterizing in vivo glenohumeral joint contact patterns during dynamic activities. This approach overcomes limitations associated with cadaveric experiments and static imaging techniques. Future research efforts will use this experimental approach to objectively assess the glenohumeral joint contact patterns in asymptomatic normal individuals and those with pathologic conditions affecting the shoulder.

\section{Acknowledgments}

This project was supported by grant number AR051912 from NIH/NIAMS.

\section{References}

[1] J. Yu, M. H. McGarry, Y.-S. Lee, L. V. Duong, and T. Q. Lee, "Biomechanical effects of supraspinatus repair on the glenohumeral joint," Journal of Shoulder and Elbow Surgery, vol. 14, no. 1, supplement 1, pp. S65-S71, 2005.

[2] R. Gupta and T. Q. Lee, "Positional-dependent changes in glenohumeral joint contact pressure and force: possible biomechanical etiology of posterior glenoid wear," Journal of Shoulder and Elbow Surgery, vol. 14, no. 1, supplement 1, pp. S105-S110, 2005.

[3] P. E. Greis, M. G. Scuderi, A. Mohr, K. N. Bachus, and R. T. Burks, "Glenohumeral articular contact areas and pressures following labral and osseous injury to the anteroinferior quadrant of the glenoid," Journal of Shoulder and Elbow Surgery, vol. 11, no. 5, pp. 442-451, 2002.

[4] H. Graichen, S. Hinterwimmer, R. von Eisenhart-Rothe, T. Vogl, K.-H. Englmeier, and F. Eckstein, "Effect of abducting and adducting muscle acitivity on glenohumeral translation, scapular kinematics and subacromial space width in vivo," Journal of Biomechanics, vol. 38, no. 4, pp. 755-760, 2005.

[5] S. Hinterwimmer, R. von Eisenhart-Rothet, M. Siebert, et al., "Influence of adducting and abducting muscle forces on the subacromial space width," Medicine and Science in Sports and Exercise, vol. 35, no. 12, pp. 2055-2059, 2003.

[6] G. P. Pappas, S. S. Blemker, C. F. Beaulieu, T. R. McAdams, S. T. Whalen, and G. E. Gold, "In vivo anatomy of the Neer and Hawkins sign positions for shoulder impingement," Journal of Shoulder and Elbow Surgery, vol. 15, no. 1, pp. 40-49, 2006.

[7] C. M. L. Werner, D. Weishaupt, S. Blumenthal, A. Curt, P. Favre, and C. Gerber, "Effect of experimental suprascapular nerve block on active glenohumeral translation in vivo," Journal of Orthopaedic Research, vol. 24, no. 3, pp. 491-500, 2006.

[8] H. Graichen, T. Stammberger, H. Bonel, E. Karl-Hans, M. Reiser, and F. Eckstein, "Glenohumeral translation during active and passive elevation of the shoulder-a 3D open-MRI study," Journal of Biomechanics, vol. 33, no. 5, pp. 609-613, 2000.

[9] M. Mahfouz, G. Nicholson, R. Komistek, D. Hovis, and M. Kubo, "In vivo determination of the dynamics of normal, rotator cuff-deficient, total, and reverse replacement shoulders," Journal of Bone and Joint Surgery A, vol. 87, supplement 2, pp. 107-113, 2005.

[10] C. W. A. Pfirrmann, M. Huser, G. Szekely, J. Hodler, and C. Gerber, "Evaluation of complex joint motion with computerbased analysis of fluoroscopic sequences," Investigative Radiology, vol. 37, no. 2, pp. 73-76, 2002.

[11] I. S. Talkhani and C. P. Kelly, "Movement analysis of asymptomatic normal shoulders: a preliminary study," Journal of Shoulder and Elbow Surgery, vol. 10, no. 6, pp. 580-584, 2001. 
[12] D. G. Mandalidis, B. S. Mc Glone, R. F. Quigley, D. McInerney, and M. O'Brien, "Digital fluoroscopic assessment of the scapulohumeral rhythm," Surgical and Radiologic Anatomy, vol. 21, no. 4, pp. 241-246, 1999.

[13] J.-P. Baeyens, P. Van Roy, A. De Schepper, G. Declercq, and J.-P. Clarijs, "Glenohumeral joint kinematics related to minor anterior instability of the shoulder at the end of the late preparatory phase of throwing," Clinical Biomechanics, vol. 16, no. 9, pp. 752-757, 2001.

[14] I. K. Y. Lo and S. S. Burkhart, "Double-row arthroscopic rotator cuff repair: re-establishing the footprint of the rotator cuff," Arthroscopy, vol. 19, no. 9, pp. 1035-1042, 2003.

[15] S. Tashman and W. Anderst, "In-vivo measurement of dynamic joint motion using high speed biplane radiography and CT: application to canine ACL deficiency," Journal of Biomechanical Engineering, vol. 125, no. 2, pp. 238-245, 2003.

[16] M. J. Bey, R. Zauel, S. K. Brock, and S. Tashman, "Validation of a new model-based tracking technique for measuring threedimensional, in vivo glenohumeral joint kinematics," Journal of Biomechanical Engineering, vol. 128, no. 4, pp. 604-609, 2006.

[17] G. Wu, F. C. T. van der Helm, H. E. J. Veeger, et al., "ISB recommendation on definitions of joint coordinate systems of various joints for the reporting of human joint motion-part II: shoulder, elbow, wrist and hand," Journal of Biomechanics, vol. 38, no. 5, pp. 981-992, 2005.

[18] A. R. Karduna, P. W. McClure, L. A. Michener, and B. Sennett, "Dynamic measurements of three-dimensional scapular kinematics: a validation study," Journal of Biomechanical Engineering, vol. 123, no. 2, pp. 184-190, 2001.

[19] J. J. Crisco, D. C. Moore, G. E. Marai, et al., "Effects of distal radius malunion on distal radioulnar joint mechanics—an in vivo study," Journal of Orthopaedic Research, vol. 25, no. 4, pp. 547-555, 2007.

[20] G. E. Marai, D. H. Laidlaw, C. Demiralp, S. Andrews, C. M. Grimm, and J. J. Crisco, "Estimating joint contact areas and ligament lengths from bone kinematics and surfaces," IEEE Transactions on Biomedical Engineering, vol. 51, no. 5, pp. 790799, 2004.

[21] G. A. Ateshian, S. D. Kwak, L. J. Soslowsky, and V. C. Mow, "A stereophotogrammetric method for determining in situ contact areas in diarthrodial joints, and a comparison with other methods," Journal of Biomechanics, vol. 27, no. 1, pp. 111-124, 1994.

[22] R. Kelkar, V. M. Wang, E. L. Flatow, et al., "Glenohumeral mechanics: a study of articular geometry, contact, and kinematics," Journal of Shoulder and Elbow Surgery, vol. 10, no. 1, pp. 73-84, 2001.

[23] L. J. Soslowsky, E. L. Flatow, L. U. Bigliani, R. J. Pawluk, G. A. Ateshian, and V. C. Mow, "Quantitation of in situ contact areas at the glenohumeral joint: a biomechanical study," Journal of Orthopaedic Research, vol. 10, no. 4, pp. 524-534, 1992.

[24] J. J. P. Warner, M. K. Bowen, X.-H. Deng, J. A. Hannafin, S. P. Arnoczky, and R. F. Warren, "Articular contact patterns of the normal glenohumeral joint," Journal of Shoulder and Elbow Surgery, vol. 7, no. 4, pp. 381-388, 1998.

[25] R. A. Creighton, B. J. Cole, G. P. Nicholson, A. A. Romeo, and E. P. Lorenz, "Effect of lateral meniscus allograft on shoulder articular contact areas and pressures," Journal of Shoulder and Elbow Surgery, vol. 16, no. 3, pp. 367-372, 2007.

[26] L. E. DeFrate, H. Sun, T. J. Gill, H. E. Rubash, and G. Li, "In vivo tibiofemoral contact analysis using 3D MRI-based knee models," Journal of Biomechanics, vol. 37, no. 10, pp. 14991504, 2004.
[27] L. J. Soslowsky, E. L. Flatow, L. U. Bigliani, and V. C. Mow, "Articular geometry of the glenohumeral joint," Clinical Orthopaedics and Related Research, no. 285, pp. 181-190, 1992.

[28] J. Hodler, R. A. Loredo, C. Longo, D. Trudell, J. S. Yu, and D. Resnick, "Assessment of articular cartilage thickness of the humeral head: MR-anatomic correlation in cadavers," American Journal of Roentgenology, vol. 165, no. 3, pp. 615620, 1995.

[29] H. Graichen, J. Jakob, R. von Eisenhart-Rothe, K.-H. Englmeier, M. Reiser, and F. Eckstein, "Validation of cartilage volume and thickness measurements in the human shoulder with quantitative magnetic resonance imaging," Osteoarthritis and Cartilage, vol. 11, no. 7, pp. 475-482, 2003.

[30] L. Yeh, S. Kwak, Y.-S. Kim, et al., "Evaluation of articular cartilage thickness of the humeral head and the glenoid fossa by MR arthrography: anatomic correlation in cadavers," Skeletal Radiology, vol. 27, no. 9, pp. 500-504, 1998. 